\title{
PATIENT SATISFACTION AND MARGINAL BONE LOSS OF DIFFERENT TREATMENT MODALITIES OF MANDIBULAR KENNEDY CLASS II CASES
}

\author{
Shaimaa Lotfy * and M Shady Nabhan **
}

\begin{abstract}
Objectives: This research was carried out to evaluate patient satisfaction and marginal bone loss of different treatment modalities (using double OT attachment, double OT with posterior implant and conventional partial denture) of Kennedy class II cases.

Materials and Methods: Totally, twenty-one patients with unilateral distal extension partially edentulous lower arches were randomly assigned into three groups (Group A, B\&C). Group (A): patients were rehabilitated with removable partial denture retained by unilateral double OT attachment. Group (B): patients were rehabilitated with removable partial denture retained by unilateral double OT attachment and a dental implant placed in a second molar area with $3.7 \mathrm{~mm}$ diameter and $10 \mathrm{~mm}$ length. Group (C): patients were rehabilitated with a conventional removable partial denture. Patients were selected with following criteria: Patients with mandibular Kennedy class II with the second premolar as last standing abutment, The remaining teeth should be free from periodontal diseases , Patients should exhibitied sufficient interarch space, with healthy supporting structures and abutments with 1:1.5 crown root ratio, and should have sufficient bone height $>$ $12 \mathrm{~mm}$ and bone width $>6 \mathrm{~mm}$ for implant placement. In this study, marginal bone height changes around premolar teeth were evaluated at the time of prostheses insertion, six months and one year later and patient satisfaction was evaluated one month after insertion.
\end{abstract}

Results: Insignificant difference was detected between the three groups at the different followup intervals, regarding marginal bone loss. After one year follow up, bone loss was $0.17 \pm 0.04$, $0.14 \pm 0.054 \mathrm{~mm}$ for group $A, 0.09 \pm 0.05,0.11 \pm 0.043 \mathrm{~mm}$ for group $B$ and $0.15 \pm 0.034,0.13 \pm 0.044$ $\mathrm{mm}$ for group $\mathrm{C}$ on the distal and mesial sides of the second premolar respectively. On the other hand, regarding ease of cleaning, group $\mathrm{C}$ showed higher median value (86) compared to group A (64) and group B (51) and Kruskal Wallis test showed sig difference between group C and group B. Overall patient satisfaction and comfort during eating group A and group B showed statistically significant higher median value compared to group $\mathrm{C}$ and Kruskal Wallis test showed signigcant difference between group $\mathrm{C}$ and other groups.

* Lecturer of Removable Prosthodontics, Faculty of Dentistry Ain Shams University.

** Lecturer of Removable Prosthodontics, Faculty of Dentistry Ain Shams University. 
Conclusion: Within the limitations of this study, it could be concluded that for unilateral partially edentulous patients, all the treatment modalities constructed in this study are reliable options regarding marginal bone loss, but for overall patient satisfaction and comfort with eating, unilateral partial dentures retained by double OT attachment with or without posterior implant are better than conventional removable partial dentures however regarding ease of cleaning conventional partial dentures showed better results.

Key Words: Kennedy classII, double OT, attachment, dental implant, marginal bone height and patient satisfaction.

\section{INTRODUCTION}

Removable partial dentures remain an essential prosthetic consideration in many conditions of oral rehabilitation, especially when the edentulous spaces posterior to the remaining teeth are to be restored. Functional successful prosthetic rehabilitation requires careful attention and meticulous treatment planning. Several treatment options have been introduced to control stresses induced by distal extension bases, among which is recording of the supportive form of the edentulous areas, mesial placement of the supporting rests, using retainers exhibiting stress releasing action and recently using osseointegrated implants as posterior artificial abutments. ${ }^{(1)}$

Attachment retained removable partial denture is a viable treatment alternative through which a significant number of patients could be benefited. An attachment is a connector consisting of two or more components. One component is connected to a root, tooth or implant and the other component of the prosthesis. ${ }^{(2)}$

Although the attachment retained removable partial denture (RPD) is not used as widely as the clasp- retained type, it is not an outdated treatment modality in dentistry. Rates of failed treatment for clasp retained RPDs range from $3 \%$ to $40 \%$ with mean being $26.1 \%$ Studies have shown a success rates of $83.35 \%$ for five years, $67.3 \%$ in 15 years, and of $50 \%$ when followed up 20 years for attachment-retained cast partial dentures (CPD).$^{(3)}$ Meticulous care in treatment planning and designing of attachments should be followed to facilitate proper force distribution, retention, and stability of the removable prosthesis and adequate plaque control. Attachment selection for removable dental prosthesis mainly depends on the presence of remaining tooth structure, the intra- and inter-maxillary relationships, aesthetics, availability in the market, ease of utilization, low technical expertise, and serviceability. ${ }^{(4)}$ Extra coronal attachments are preferred and are considered more efficient in providing retention and restoring function and aesthetics over intra coronal attachments as the latter obviate the risk for over contouring of distal portion of the crown, which can result in periodontal breakdown as a result of increased plaque collection..$^{(5-7)}$

RHEIN OT Cap (Extracoronal castable semiprecision attachment) is a resilient distal extension attachment. It is indicated to be used with removable partial dentures. OT Cap functions as a stabilizing retentive connector. Also, for treatment plans which require stress releasing action, OT Cap provides a "Cushion Effect" similar to a shock absorber. ${ }^{(8)}$

The use of oral implants for the rehabilitation of partial edentulism is now a well-accepted treatment modality ${ }^{(9,10)}$. The survival of implant restorations in partially edentulous patients has been within ranges similar to those for implant survival in totally edentulous patients ${ }^{(11)}$.

The use of dental implants as distal abutments can convert distal extension removable partial dentures from tooth-tissue supported prostheses to tooth- implant supported and/or retained 
prostheses. A posteriorly placed implant provides a definite stop stability and eliminates the problems often associated with tooth-tissue supported distal extension removable partial dentures ${ }^{(12)}$.

For patients with unilateral edentulism in molar region, removable partial dentures with a unilaterally designed framework claimed to be more comfortable during mastication and speech, and more profound effect is anticipated on patients' acceptance due to its relative simplicity. However, clinical use of the unilaterally designed framework is criticized owing to the poor retention and stability and difference in effect on the supporting structure compared to the removable partial denture with bilaterally designed frameworks ${ }^{(13)}$

However, a question remains will the use of unilateral partial dentures retained by double OT attachment or double OT with posterior implant produce equivalent patient satisfaction and marginal bone loss as conventional partial dentures for patients with mandibular Kennedy class II?

\section{MATERIALS AND METHODS}

Twenty-one patients with unilateral distal extension partially edentulous lower arches were selected to share in this study from out-patients clinic of the Prosthodontic Department, Faculty of Dentistry, Ain Shams University. Patients were selected with the following criteria: Patients with mandibular Kennedy class II with the second premolar as last standing abutment, The remaining teeth should be free from periodontal diseases. Patients were exhibiting sufficient interarch space, Good oral hygiene, with healthy supporting structures and abutments with 1:1.5 crown root ratio, Patients should be free from any systemic diseases that may lead to bone resorption, patient should had sufficient bone height $>12 \mathrm{~mm}$ and bone width $>6 \mathrm{~mm}$ for implant placement.

Intraoral examination included visual and digital assessment of oral hygiene and clinical condition of remaining teeth and residual ridge. Cone Beam CT was taken to detect the presence of any pathological lesions, cysts or remaining roots in the lower ridge and to evaluate the condition of the abutments \& to determine optimum placement site of the posterior implant.

Maxillary and mandibular diagnostic casts were mounted on a semi-adjustable articulator using a tentative jaw relation and maxillary face bow record. This was important to evaluate the interarch space, and to assess the jaw relation. An interarch space should be at least $7 \mathrm{~mm}$ to accommodate for the male and female attachment parts, artificial teeth and minimum space of $1 \mathrm{~mm}$ for hygiene beneath the attachment. Also, $10 \mathrm{~mm}$ inter occlusal area in $2^{\text {nd }}$ molar area was essential to accommodate the space for ball and socket attachment over the dental implant

Patients were divided randomly into three equal groups; each group including seven patients: Group (A): patients were rehabilitated with removable partial dentures retained by unilateral double OT attachment. Group (B): patients were rehabilitated with removable partial dentures retained by unilateral double OT attachment and a dental implant placed in the molar area. Group (C): patients were rehabilitated with conventional removable partial dentures.

\section{1) Construction of unilateral double OT attach- ment retained removable partial denture (group A):}

The first and second premolars of the edentulous side were prepared with sufficient occlusal and labial reduction with shoulder finishing lines circumferentially to receive two-unit veneered crowns. The gingiva of the bridge abutments was retracted using a retraction cord, which was left for few minutes then, removed immediately before impression making. The impression was made using rubber base impression material (Zhermack zetaplus 
rubber base - Italy) then poured in improved stone to obtain the master cast. The impression was sent to the laboratory to make metal try-in as two splinted crowns with the double OT attachment attached to the metal of lower second premolar crown. The metal try-in was examined intraorally to detect any open margins, then the appropriate shade for the artificial teeth was selected then the assembly was sent to the laboratory for the addition of porcelain facing. Pick up impression for attachment assembly was made with putty rubber base impression in an acrylic resin special tray painted with adhesive to pick up the assembly and record the edentulous span. Impressions were poured with stone plaster to obtain master casts. The master cast was duplicated using Agar- Agar hydrocolloid duplicating material in the duplicator to produce the refractory cast. Readymade wax pattern was used to fabricate the partial denture framework. The denture base meshwork was spaced around the position of the attachment. The framework was cast in Cobalt-Chromium alloy and tried in the patient's mouth and adjusted.

The upper cast was mounted on the semiadjustable articulator using maxillary face bow. The lower cast with the framework was mounted on the articulator using centric occluding relation record. For setting up modified anatomic acrylic resin artificial teeth with 20 degrees following the lingualized occlusal concept. The partial denture with waxed try-in was tried in the patient's mouth, and jaw relation was rechecked. The partial denture was flasked, packed, cured, finished and polished.

The self-cured acrylic resin (Reliance DuraLay self-cured acrylic resin) used for the technique of direct pick up was methylmethaacrylate-free. The female part of the OT attachment was placed on the male part intraorally. The fitting surface of the acrylic denture was hollowed in the area of the attachment. Then the denture was inserted in place with self-cured acrylic resin in the hollow (the patient was biting in centric position) and left to cure to pick up the female part in its fitting surface. The finished partial denture was refitted in the patient's mouth and checked for retention, stability, extension, and occlusion. The occlusion was equilibrated to achieve a stable occlusal contact in centric occlusion. (Fig 1)

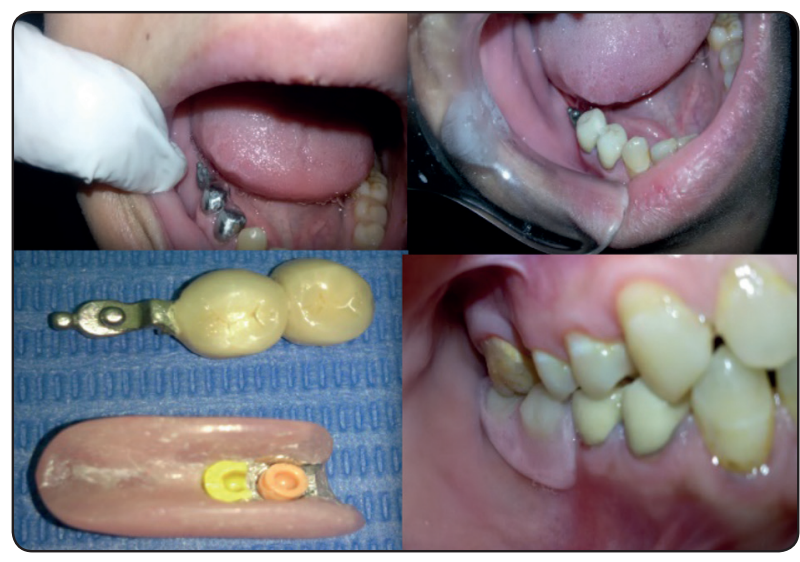

Fig (1) Stages of construction of unilateral double OT attachment

2) Construction of unilateral double OT attachment retained removable partial denture with posterior implant (group B):

Diagnostic cone beam computerized tomography (CBCT) was made for every patient to determine the approximate bone width and height at the proposed implant site. Each patient received one implant in the second molar area with dimensions $10 \mathrm{~mm}$ length and $3.7 \mathrm{~mm}$ width (Implant direct, Replant тм implant, CA 91301 Germany). The surgical procedures were done following "two stages submerged surgical protocol." Three months later, the implant was exposed, and healing abutments were screwed in place for two weeks to improve healing. Ball attachment was attached to implant and socket was picked up on the fitting surface of removable partial denture using self-cure acrylic resin at the same time of pick-up of OT attachment.

The double OT attachment was constructed for all patients similar to group A. Finally, pick-up of the OT attachment and attachment of the implant 
was done. Areas in the lower partial denture opposing to the inserted implant and OT attachment were marked and relieved on the fitting surfaces of the denture. The denture was placed in the patient's mouth to check and ensure complete seating and proper intercuspal relation. Acrylic pickup material was added to the relieved areas, and the denture was reseated inside the patient's mouth. The excess acrylic resin was removed. Recall appointments were scheduled for patients for evaluation of the prosthesis and to perform any needed adjustments.

(Fig 2)

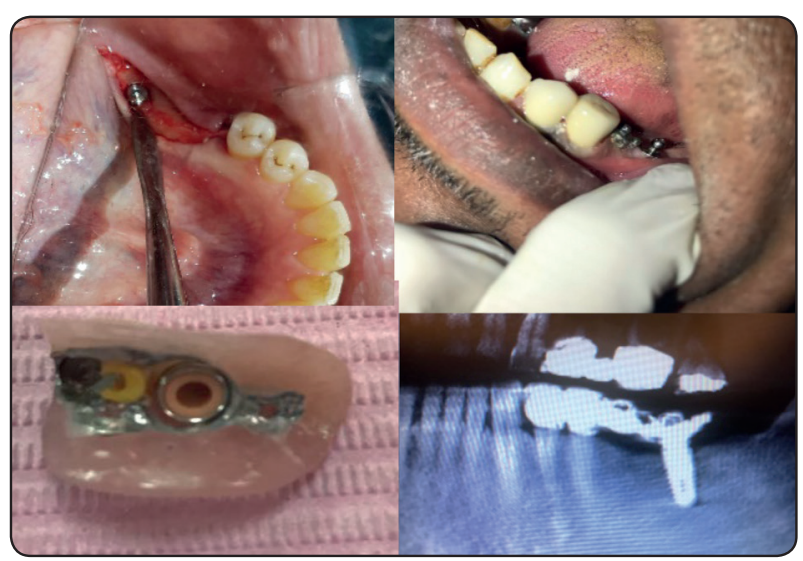

Fig (2) Stages of construction of unilateral double OT attachment with posterior implant in the second molar area

\section{3) Construction of conventional removable partial denture (group $C)$ :}

For patients of this group, conventional removable partial dentures were constructed as follows:

Saucer shaped rest seat preparations were prepared on the abutments far from the edentulous areas, the floor of the seat was inclined toward the center of the tooth. The secondary impression was made for the lower arch using rubber base material and poured in stone to produce the master cast. The master cast was finally surveyed. Undesirable undercuts were blocked-out using block out wax and trimmed parallel to the path of insertion.

Refractory casts were obtained by duplicating the modified master casts using agar-agar hydrocolloid duplicating material. Partial denture framework was constructed using ready-made wax, the components of the framework were combined denture base, lingual bar major connector, double Aker clasp on the second premolar and first molars on the intact side; combination clasp having gingivally approaching clasp arm (I bar) on the last standing abutment on the edentulous side \& cingulum rest on the intact side. Cast procedures were completed, and metallic framework was obtained. The metallic cobalt chromium framework was verified in the patient mouth to test the fit of the occlusal rests and proper location of the major connector and clasps.

Altered cast impression technique was done after checking the metal framework, Centric occluding relation was made to mount the upper and lower casts for setting up modified anatomic acrylic resin artificial teeth with 20 degrees cusp angle following the lingualized occlusal concept. The waxed up denture was tried in the patient's mouth. Denture processing was carried out in the usual manner. The finished denture was delivered to the patient after performing the needed occlusal adjustments. (Fig 3)

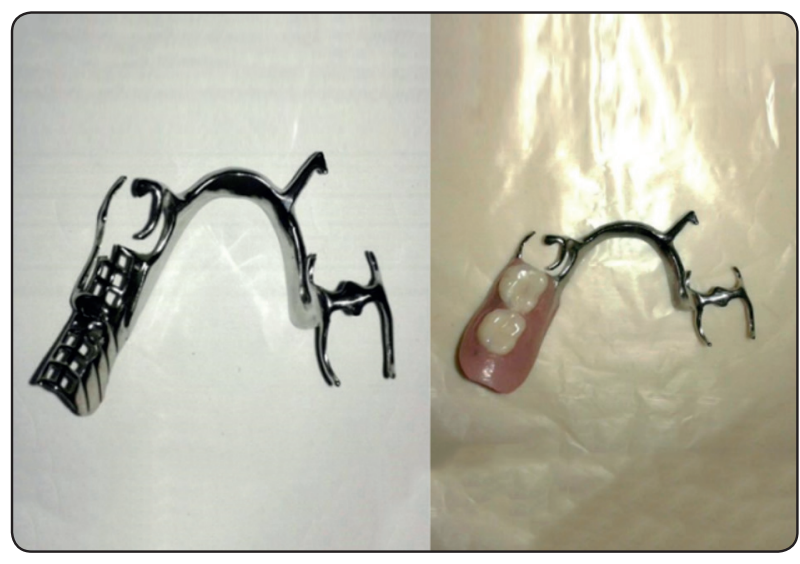

Fig (3) Finished metallic framework \& final partial denture 


\section{Assessed outcomes:}

\section{a-Radiographic evaluation}

Marginal bone height change around the second premolar was evaluated using the linear measurement system supplied by the digital parallel radiographic technique. Marginal bone height changes around abutments were monitored. The marginal bone loss at different intervals was obtained by calculating the difference in bone height at that interval from the baseline measurement. The measurements were carried out at the end of each follow-up appointment (at insertion, 6, and 12 months post insertion).

\section{b-Patient satisfaction}

Patients specified their level of satisfaction by indicating a position along the continuous line of a $100 \mathrm{~mm}$ visual analog scale (VAS). ${ }^{(14)}$ The VAS line represents a spectrum of feelings between two extremes, with vertical mark on the line representing their feelings at that time. ${ }^{(15)}$ The VAS was intervieweradministered. The patients rated their dentures in terms of; ease of cleaning, comfort while eating and overall satisfaction. Rating of each category used keywords "not at all satisfied" at $0 \mathrm{~mm}$ and "extremely satisfied" at $100 \mathrm{~mm}$. A pencil mark was made across the horizontal line at a point corresponding to the subjective feeling of the subject. Satisfaction was then measured as the distance in millimeters from the left end limit to the point of the pencil mark representing the VAS score of the subject. The higher the score, the better the prosthesis as perceived by the subject. ${ }^{(16)}$ Patient satisfaction was examined 1 month after delivery.

\section{Statistical analysis}

Numerical data were explored for normality by checking the data distribution, calculating the mean and median values, evaluating histograms and normality curves and using KolmogorovSmirnov and Shapiro-Wilk tests. Parametric Data were presented by mean, standard deviation (SD) and nonparametric data presented as a median and interquartile range. One-way ANOVA and its nonparametric alternative (Kruskal Wallis test) were used for comparison between groups followed by Tukey post hock test and Mann Whitney test with Bonferroni correction for nonparametric data. The significance level was set at $\mathrm{P} \leq 0.05$. Statistical analysis was performed with IBM ${ }^{\circledR}$ SPSS $^{\circledR}$ Statistics Version 20 for Windows. Results were represented in tables (1-3)

\section{RESULTS}

TABLE (1) The mean (mm), standard deviation (SD) values and comparison between amounts of bone changes distal to the second premolar in the three groups during follow up periods using one -way ANOVA test.

\begin{tabular}{|c|c|c|c|c|c|c|c|}
\hline \multirow{2}{*}{$\begin{array}{c}\text { Bone change distal to } \\
2^{\text {nd }} \text { premolar }\end{array}$} & \multicolumn{2}{|c|}{ Group A } & \multicolumn{2}{c|}{ Group B } & \multicolumn{2}{c|}{ Group C } & P value \\
\cline { 2 - 8 } & Mean & Std. deviation & Mean & Std. deviation & Mean & Std. deviation \\
\hline Insertion- 6 months & 0.08 & 0.01 & 0.045 & 0.03 & 0.043 & 0.015 & 0.178 \\
\hline 6-12 months & 0.09 & 0.005 & 0.08 & 0.04 & 0.11 & 0.03 & 0.468 \\
\hline Insertion- 12months & 0.17 & 0.04 & 0.12 & 0.05 & 0.15 & 0.034 & 0.09 \\
\hline
\end{tabular}


TABLE (2) The mean (mm) ,standard deviation (SD) values and comparison between amounts of bone changes mesial to the second premolar in the three groups during follow up periods using one -way ANOVA test.

\begin{tabular}{|c|c|c|c|c|c|c|c|}
\hline \multirow{2}{*}{$\begin{array}{c}\text { Bone change mesial to } 2^{\text {nd }} \\
\text { premolar }\end{array}$} & \multicolumn{2}{|c|}{ Group A } & \multicolumn{2}{|c|}{ Group B } & \multicolumn{2}{|c|}{ Group C } & \multirow{2}{*}{$P$ value } \\
\hline & Mean & Std. deviation & Mean & Std. deviation & Mean & Std. deviation & \\
\hline Insertion- 6 months & 0.023 & 0.01 & 0.04 & 0.023 & 0.045 & 0.04 & 0.178 \\
\hline 6-12 months & 0.13 & 0.04 & 0.075 & 0.05 & 0.09 & 0.035 & 0.468 \\
\hline Insertion- 12 months & 0.15 & 0.054 & 0.11 & 0.043 & 0.13 & 0.044 & 0.117 \\
\hline
\end{tabular}

As confirmed in tables $1 \& 2$ throughout the whole follow up period there was statistically insignificant difference between the three groups with the least mean difference within group C.

TABLE (3) Satisfaction scores for all groups.

\begin{tabular}{|c|c|c|c|c|c|c|c|}
\hline \multirow{2}{*}{$\begin{array}{c}\text { Patient satisfaction } \\
\text { 1months }\end{array}$} & \multicolumn{2}{|c|}{ Group A } & \multicolumn{2}{c|}{ Group B } & \multicolumn{2}{c|}{ Group C } & \multirow{2}{*}{ P value } \\
\cline { 2 - 7 } & Median & IQR & Median & IQR & Median & IQR & \\
\hline Ease of cleaning & $64^{\text {ab }}$ & 48 & $51^{\mathrm{b}}$ & 34 & $86^{\mathrm{a}}$ & 31 & $<0.05$ \\
\hline Comfort while eating & $87^{\mathrm{a}}$ & 28 & $93^{\mathrm{a}}$ & 30 & 68 & 33 & $<0.05$ \\
\hline Overall satisfaction & $81^{\mathrm{a}}$ & 33 & $85^{\mathrm{a}}$ & 45 & 63 & 32 & $<0.05$ \\
\hline
\end{tabular}

Kruskal Wallis test followed by Mann Whitney test with Bonferroni correction were done. Similar superscript letters indicate insignificant statistical difference.

Regarding ease of cleaning group C showed highest value followed by group A followed by group B and Kruskal Wallis test showed significant statistical difference, however, post hock test showed a significant difference between group $\mathrm{C}$ and group $\mathrm{B}$ and insignificant difference between group $\mathrm{A}$ and group $\mathrm{C}$ and between group $\mathrm{A}$ and group $\mathrm{B}$.

Regarding comfort while eating group B showed highest value followed by group A then group C. Kruskal Wallis test showed statistically significant difference and post hock test showed significant difference between group $\mathrm{C}$ and other groups. Regarding overall satisfaction group B showed highest value followed by group A followed by group C and Kruskal Wallis test showed significant statistical difference, however, post hock test showed insignificant difference between group $\mathrm{C}$ and other groups and insignificant difference between group A and group B.

\section{DISCUSSION}

Management of unmodified lower Kennedy class II sometimes becomes challenging as treatment with conventional partial denture needs extension to the dentulous side to avoid the risk of swallowing the prosthesis and to provide sufficient stability. However, this prosthesis may cause discomfort to the patient. Unilateral double OT attachment provides a solution for this problem as it provides sufficient retention and stability to retain the unilateral removable partial denture. However, there is a risk of causing excessive forces to abutment teeth. Dental implants may provide a solution to this protentional problem. So, in this study, evaluation of the effect of those 3 treatment options regarding bone changes around abutment teeth and patient satisfaction were conducted.

Bone changes around the abutments were evaluated with intraoral digital parallel radiographic 
technique. Parallel radiographic technique has the advantages of less magnification, and a real relationship between the bone height and the teeth was demonstrated, digital radiography offers many advantages over conventional radiography. It eliminates the need for film and film developing, and it allows for lower radiation exposure. Bone changes were evaluated around the abutment (mesial and distal to the last abutment). ${ }^{(17,18)}$

Results of this study showed statistically insignificant difference between groups regarding bone changes around abutment teeth and all treatment options had minimal bone changes around abutment (less than $0.2 \mathrm{~mm}$ ). This may be due to the use of stress releasing clasp with conventional partial denture or using resilient extra coronal attachment as it was reported that the least stress was observed when using a non-rigid connector, while rigid connectors in such situations cause higher stresses in the supporting structure. ${ }^{(19)}$

The use of removable partial dental prosthesis associated with a dental implant to convert Kennedy class II to class III dental prosthesis decrease stresses around abutment teeth. These may also explain the decrease in bone changes around abutment teeth in group B. ${ }^{(20)}$

Patient satisfaction was measured at one month to give sufficient time for patient adaptation for new prostheses and settling of the prosthesis. It was evaluated regarding ease of cleaning, comfort while eating, and overall satisfaction. This study showed better cleaning on prosthesis with conventional partial denture compared to extra-coronal attachment and dental implant, and this may be explained by the presence of extra-coronal attachment, and dental implant may complicate oral hygiene measures. It has been reported that one of the main causes of attachment retained partial denture failure is lack of proper oral hygiene. ${ }^{(21)}$

In this study regarding comfort while eating, group A, B showed better results compared to group
$\mathrm{C}$ and the difference was statistically significant. Comfort while eating may be enhanced with extracoronal attachment and dental implant as it converts condition from tooth- tissue supported to toothimplant supported. ${ }^{(22)}$

Patient overall satisfaction may be improved with the use of a dental implant and double OT attachment due to enhanced retention, support, and stability and reduced bulk of prosthesis. ${ }^{(23)}$

\section{CONCLUSION}

Within the limitations of this study, it could be concluded that for unilateral partially edentulous patients, all the treatment modalities constructed in this study are reliable options regarding marginal bone loss, but for overall patient satisfaction and comfort with eating, unilateral partial dentures retained by double OT attachment with or without posterior implant are better than conventional removable partial dentures however regarding ease of cleaning, conventional partial dentures showed better results.

\section{REFERENCE}

1. Monteith B. Management of loading forces on mandibular distal- extension prosthesis. Part 1: Evaluation of concepts for design. J. Prosthet. Dent 1984; 52: 673.

2. Preiskel HW.Precision Attachment in Prosthodontics. Henry Kimpton Pub. London ,69-78, 1995.

3. Owal B. Precision attachment retained removable partial dentures: Part1. Technical long-term study. Int J Prosthodont 1991;4(3):249- 57.

4. Nicola U, Zitzmann, Rohner URS, Weiger R, Gabriel K. When to choose which retention element to use for removable dental prosthesis. Int J Prosthodont 2009; 22:161-67.

5. Awang RAR, Arief EM, Hassan A. Spring loaded plunger attachment for retention of removable partial denture: A case report. Arch Orofac Sci 2008; 3:32-5.

6. Chikunov I, Doan P, Vahidi F. Implant-retained partial overdenture with resilient attachments. J Prosthodont 2008; 17:141-8 
7. Jenkins G. Precision Attachments: A Link to Successful Restorative Treatment. Quintessence Publishing; 1999:11

8. Ashish R. J., Jacob M. P., Padma A. Attachment retained unilateral distal extension (Kennedy's Class II modification I) Cast partial denture, a case report, Int J Prosthodont Rest Denti 2012 ,2; 101-107.

9. Lindquist L.W., Carlsson G.E., and Jemt T. A prospective 15-year follow-up study of mandibular fixed prostheses supported ossointegrated implants. Clin Oral Implants Res. 1996, 7; 329.

10. Remeo E., Chiapasco M., Ghisolfi M., and Vogel G. Longterm clinical effectiveness of oral implants in the treatment of partial edentulism seven-year life table analysis of a prospective study with ITI dental implants system for single tooth restorations. Clin Oral Implants Res.2002, 13; 133.

11. Mufu A., and Champ R.J. Replacing posterior teeth with free standing implants, Four-year Prosthodontic results of a prospective study. JADA. 1998, 129; 1097.

12. Carr AB, Brown DT. McCracken's removable partial prosthodontics. 12 $2^{\text {th }}$ ed. St Louis: CV Mosby; 24, 2011.

13. Makkar S., Chhabra A., and Khare A. "Attachment retained Removable partial denture: a case report, Int J Clinic Dent Science. 2011, 2; 39-43.

14. Aragon CE, Cornacchio AL, Ibarra LM, Saad MN, Zibrowski E. Implant overdentures: Dental students' performance in fabrication, denture quality, and patient satisfaction. J Dent Educ 2010; 74:993-1001
15. Petricevic N, Celebic A, Rener-Sitar K. Improvement of patient's satisfaction and oral health related quality of life by the implant and prosthodontic treatment; 2007.

16. Akinyamoju CA, Ogunrinde TJ, Taiwo JO, Dosumu OO, Nigerian. Comparison of Patient Satisfaction with Acrylic and Flexible Partial Dentures Postgraduate Medical Journal 2017, 24 (3) .

17. Mansour P.A., and Dudhia R.: Implant radiography and tadilogy. Australian Dental Journal. 2008, 53; 11.

18. Christensen: why switch to digital radiography? JADA. 2004, 135; 1437.

19. Nishimura RD, Ochiai KT, Caputo AA, Jeong CM. Photoelastic stress analysis of load transfer to implants and natural teeth comparing rigid and semirigid connectors. J Prosthet Dent 1999; 81:696-703.

20. Karla Z., Gizella M. Abrão,. Placement of a distal implant to convert a mandibular removable Kennedy class I to an implant-supported partial removable Class III dental prosthesis: A systematic review. Prosthet Dent 2015; 113:6.

21. Van der Bilt A. Human oral function a review Braz. J. Oral Sci.2002, 1; 7-18.

22. Mitrani R, Brudvik JS, Phillips KM. Posterior implants for distal extension removable prostheses: a retrospective study. Int J Periodontics Restorative Dent 2003; 23:353-9.

23. Budtz-Jorgensen E. Restoration of the partially edentulous mouth:A comparison of overdenture, removable partial denture, fixed partial denture and implant treatment. J Dent.1996;24:237-44. 